\title{
Electron Pairs and Excitons in Quasi-One-Dimensional Nanostructures
}

\author{
S. Bednarek, B. Szafran, T. Chwiej and J. Adamowski
}

Faculty of Physics and Nuclear Techniques

AGH University of Science and Technology

al. Mickiewicza 30, 30-059 Kraków, Poland

\begin{abstract}
A theoretical study of two-particle systems in quasi-one-dimensional quantum wires and quantum dots is presented. We have derived the analytical formula for the effective interaction potential between the charge carriers confined laterally by a strong parabolic potential and applied this formula to electron pairs in single and double quantum dots and to excitons in quantum wires. In the single quantum dot of the sufficiently large size, we have found the Wigner-type localization.
\end{abstract}

PACS numbers: 73.21.-b

\section{Introduction}

Quasi-one-dimensional nanostructures, i.e. quantum wires [1, 2] and carbon nanotubes [3] have gained a lot of attention during the last decade. A Luttinger liquid behavior $[1,3]$ and a quantization of conductance [4] have been reported. In one-dimensional (1D) nanostructures, the problem of interaction between the charge carriers is of a special importance because of the non-removable singularity of the Coulomb potential, which leads to the divergent ground state [5] in the strictly 1D exciton eigenproblem. The problem of the 1D Coulomb potential singularity can be resolved taking into account the finite-size extension of the charge carrier wave functions in the plane perpendicular to the axis of the real three-dimensional (3D) structure. The effective 1D interaction potential can be obtained by integration over the lateral degrees of freedom [6, 7]. We have succeeded [8] in performing this integration analytically and obtained a compact real-space form of the effective interaction between the charge carriers confined in quasi-1D nanostructures. In this paper, we apply this effective potential to the electron pairs and excitons in a quasi-1D environment. 


\section{Theory}

Charge carriers moving in the quasi-1D nanostructures are subjected to a strong lateral confinement potential. We assume the lateral confinement potential to be parabolic, i.e.

$$
V_{\mathrm{e}(\mathrm{h})}=\frac{1}{2} m_{\mathrm{e}(\mathrm{h})} \omega_{\mathrm{e}(\mathrm{h})}^{2}\left(x_{\mathrm{e}(\mathrm{h})}^{2}+y_{\mathrm{e}(\mathrm{h})}^{2}\right),
$$

where $x_{\mathrm{e}(\mathrm{h})}, y_{\mathrm{e}(\mathrm{h})}$ are the lateral coordinates of the electron (hole), $m_{\mathrm{e}(\mathrm{h})}$ is the electron (hole) effective mass, and $\omega_{e(h)}$ is the lateral confinement frequency for electrons (holes). If lateral confinement energies $\hbar \omega_{e}(\mathrm{~h})$ are large in comparison to the interparticle interaction energy, the lateral degrees of freedom are frozen. Then, all the physically interesting effects depend on only $z$ coordinates of the particles measured along the axis of the nanostructure. The wave function of the two-particle complex can be approximated by

$$
\Psi\left(\boldsymbol{r}_{1}, \boldsymbol{r}_{2}\right)=\psi\left(z_{1}, z_{2}\right) \phi_{\mathrm{e}}\left(x_{\mathrm{e}}, y_{\mathrm{e}}\right) \phi_{\mathrm{e}(\mathrm{h})}\left[x_{\mathrm{e}(\mathrm{h})}, y_{\mathrm{e}(\mathrm{h})}\right]
$$

The lateral single-particle ground-state wave function for the parabolic confinement has the form

$$
\phi_{\mathrm{e}(\mathrm{h})}=\exp \left[-\left(x^{2}+y^{2}\right) / 2 l_{\mathrm{e}(\mathrm{h})}^{2}\right] /\left[l_{\mathrm{e}(\mathrm{h})} \sqrt{\pi}\right],
$$

where $l_{\mathrm{e}(\mathrm{h})}=\left(\hbar / m_{\mathrm{e}(\mathrm{h})} \omega_{\mathrm{e}(\mathrm{h})}\right)^{1 / 2}$ are the oscillator lengths. The integration of the Coulomb interaction expectation value over the lateral coordinates yields the effective potential of interaction between the electron and hole separated by a distance $z$ in the $1 \mathrm{D}$ medium in the form

$$
V_{\mathrm{eff}}(z)=-\left(\frac{\pi}{2}\right)^{1 / 2} \frac{\kappa}{l} \operatorname{erfcx}\left(\frac{|z|}{2^{1 / 2} l}\right)
$$

where $l=\left[\left(l_{\mathrm{e}}^{2}+l_{\mathrm{h}}^{2}\right) / 2\right]^{1 / 2}, \kappa=e^{2} / 4 \pi \varepsilon \varepsilon_{0}, \varepsilon$ is the dielectric constant, and $\operatorname{erfcx}(x)=$ $\exp \left(x^{2}\right) \operatorname{erfc}(x)$ is the exponentially scaled complementary error function. Replacing $l_{\mathrm{h}}$ by $l_{\mathrm{e}}$ and changing the sign of the prefactor, we obtain the electron-electron effective interaction potential ( $\mathrm{cf}$. inset of Fig. 1). The derivation of effective potential (2) is based on the separability [Eq. (1)] of the two-particle wave function, which is valid for the strong lateral confinement or the spatial separation of the charge carriers. The last condition is fulfilled in the Wigner molecules and weakly coupled quantum dots, i.e. artificial molecules. Therefore, effective potential (2) is applicable not only to the quantum wires but also to the elongated quantum dots and to the weakly coupled quantum dots. We note that many authors, e.g. [7], [9], and [10], were looking for the real space form of the effective electron-electron interaction potential in the $1 \mathrm{D}$ nanostructures. So far, the effective potential was either obtained by a time-consuming numerical integration [7] or replaced by approximate analytical formulae [9, 10]. However, none of these approximate formulae reproduce the present form of potential (2) in the entire range of interparticle distance $z$. 


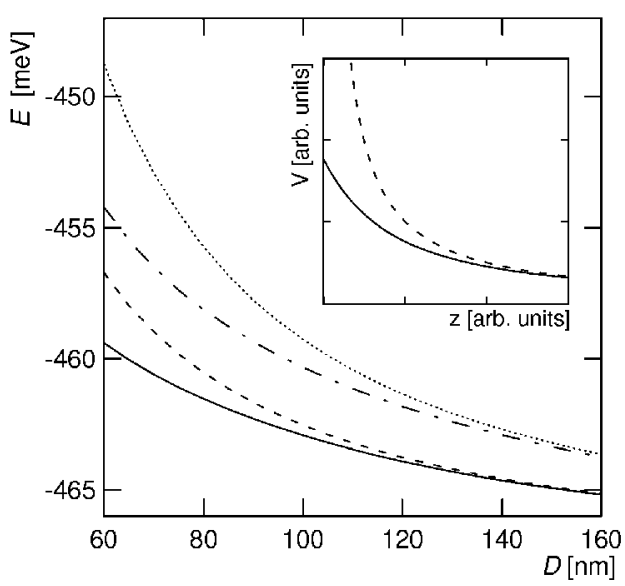

Fig. 1. The lowest energy levels of the electron pair confined in a single quantum dot with linear size $D$. The solid (dotted) line shows the energy of the even-parity singlet (triplet) state. The dashed (dash-dotted) line shows the energy of odd parity triplet (singlet) state. Inset: effective electron-electron interaction potential (solid line) and Coulomb potential (dashed line) as functions of electron-electron distance $z$.

\section{Results and discussion}

Let us first consider the electron pair confined in the single quantum dot. The two-electron Schrödinger equation for wave function $\psi\left(z_{1}, z_{2}\right)$ is solved by the imaginary time finite-difference technique. Throughout the present paper, we apply the material parameters of GaAs. Moreover, we take on $\hbar \omega_{\mathrm{e}(\mathrm{h})}=\hbar \omega=6 \mathrm{meV}$ and assume the vertical confinement to be the rectangular quantum well with depth $240 \mathrm{meV}$ and varying width $D$. Figure 1 shows that the lowest-energy levels of the confined electron pair become twofold degenerate in the limit of large $D$.

The origin of this degeneracy can be understood when looking at Fig. 2, which shows the two-electron probability density. All the states considered possess the definite spin and definite symmetry against a spatial inversion. Therefore, the two-electron probability density is invariant with respect to the interchange $z_{1} \longleftrightarrow z_{2}$, because of the definite permutation symmetry, and with respect to the reflection $\left(z_{1}, z_{2}\right) \longleftrightarrow\left(-z_{1},-z_{2}\right)$, because of the definite parity symmetry (cf. Fig. 2). We note that the two-electron probability density vanishes along the line $z_{1}=z_{2}$ for triplet states, which results from the permutational symmetry, and also along the line $z_{1}=-z_{2}$ for the even-triplet and odd-singlet states, which is caused by the simultaneous effect of the permutational and parity symmetry. When the quantum-well width increases, the electron-electron interaction contribution becomes large in comparison with the single-electron excitation energy, which leads to a creation of the Coulomb hole in the probability density for the singlet states near the line $z_{1}=z_{2}$. In consequence, for large $D$ the probability density becomes 


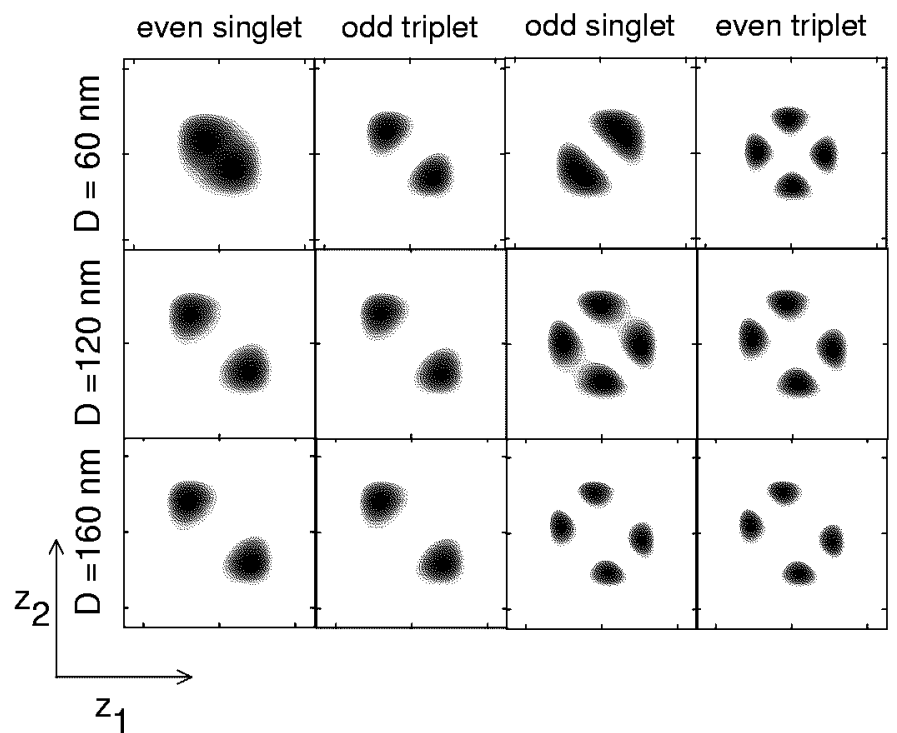

Fig. 2. Two-electron probability density $\left|\psi\left(z_{1}, z_{2}\right)\right|^{2}$ for the states with energy depicted in Fig. 1. The darker the shade of gray the larger the probability density. The length of the box sides in the first, second, and third row is 110,188 , and $250 \mathrm{~nm}$, respectively.

the same for the degenerate energy levels, which explains the degeneracy shown in Fig. 1. Moreover, Fig. 2 shows that for the quantum dots with large $D$ the electrons are spatially separated by the Coulomb repulsion, i.e. form a Wigner molecule [11].

We have applied effective interaction potential (2) to the problem of the two-electron artificial molecule [12], i.e. the electron pair confined in the two vertically coupled quantum dots. Figure 3 shows the lowest singlet and triplet energy levels of the artificial molecules as functions of thickness $b$ of the barrier separating the quantum dots. If $b$ increases, the electrons become separated and localized in the two different dots (see inset of Fig. 3). Similarly as in the case of the single quantum dot, after the charge separation takes place, the energy of the system is independent of the spin, which results in the degeneracy of the lowest singlet and triplet energy levels. When the electrons are separate, the total energy of the system exhibits the Coulombic, i.e. $\sim 1 / b$, asymptotic behavior.

The effective interaction potential (2) has also been applied to the problem of the ground state of the exciton in a quantum wire. In this case, the charge carriers are not confined in the direction of the axis of the wire. Moreover, we assume that the lateral potential is the same for the electron and the hole. Figure 4 shows the exciton binding energy defined as $E_{b}=E-2 \hbar \omega$, where $E$ is the ground-state energy of the exciton. In Fig. 4, the dashed curve shows the "exact" results, obtained by the variational approach with the original 3D Hamiltonian of the exciton, and the solid curve shows the results obtained with the $1 \mathrm{D}$ effective 


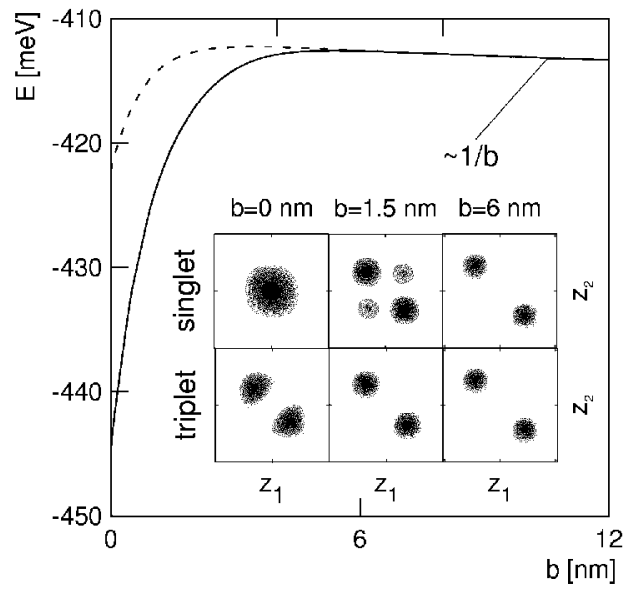

Fig. 3. The lowest singlet (solid line) and triplet (dashed line) energy levels of the electron pair in the double coupled quantum dot as functions of barrier thickness $b$. The potential-well width is $12 \mathrm{~nm}$ and depth $240 \mathrm{meV}$. Inset: two-electron probability density $\left|\psi\left(z_{1}, z_{2}\right)\right|^{2}$ for the lowest-energy singlet and triplet states. The darker the shade of gray the larger the probability density. The length of the box sides is 35,37 , and 41 $\mathrm{nm}$ for $b=0,1.5$, and $6 \mathrm{~nm}$, respectively.

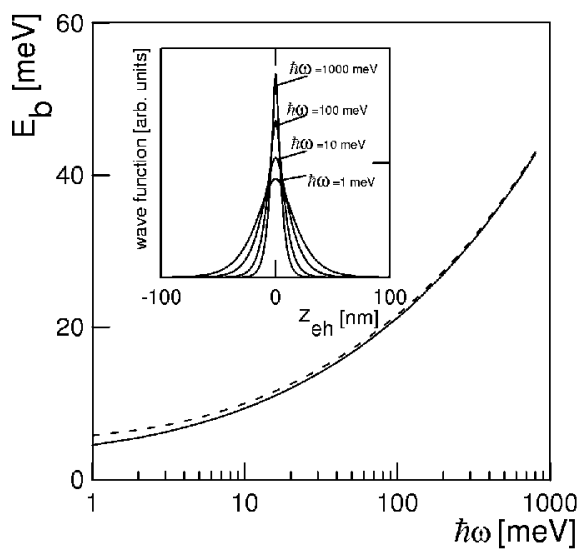

Fig. 4. Binding energy of the exciton confined in the quantum wire as a function of lateral confinement energy $\hbar \omega$. The dashed curve shows the results of the "exact" $3 \mathrm{D}$ approach and the solid curve shows the results obtained with the use of effective interaction potential (2). Inset: wave function of the exciton as a function of the relative electron-hole distance $z_{\mathrm{eh}}$, obtained with potential (2).

interaction potential (2). We see that the latter results become exact for the sufficiently strong lateral confinement. The growth of the exciton binding energy is related with the increasing localization of the relative-motion electron-hole wave 
function (cf. inset of Fig. 4), which in turn is due to the fact that the minimum of interaction potential (2) becomes deeper when the lateral confinement potential increases. The binding energy of the exciton in the quantum wire can be several times larger than that in bulk crystals.

\section{Acknowledgment}

This paper has been supported in part by the State Committee for Scientific Research.

\section{References}

[1] S. Tarucha, T. Honda, T. Saku, Solid State Commun. 94, 413 (1995).

[2] M.T. Björk, B.J. Ohlsson, T. Sass, A.I. Persson, C. Thelander, M.H. Magnusson, K. Deppert, L.R. Wallenberg, L. Samuelson, Appl. Phys. Lett. 80, 1058 (2002).

[3] A. Bachtold, C. Strunk, J.-P. Salvetat, J.-M. Bonard, L. Forro, T. Nussbaumer, C. Schonenberger, Nature 397, 673 (1999).

[4] A. Yacoby, H.L. Stormer, N.S. Wingreen, L.N. Pfeiffer, K.W. Baldwin, K.W. West, Phys. Rev. Lett. 77, 4612 (1996).

[5] R. Loudon, Am. J. Phys. 27, 649 (1959).

[6] L. Banyai, I. Galbraith, C. Ell, H. Haug, Phys. Rev. B 36, 6099 (1987).

[7] M.H. Szymanska, P.B. Littlewood, R.J. Needs, Phys. Rev. B 63, 205317 (2001).

[8] S. Bednarek, T. Chwiej, J. Adamowski, B. Szafran, Phys. Rev. B 67, 205316 (2003).

[9] R. Egger, H. Grabert, Phys. Rev. B 55, 9929 (1997).

[10] C. Fuchs, R. v. Baltz, Phys. Rev. B 63, 085318 (2001).

[11] B. Szafran, S. Bednarek, J. Adamowski, Phys. Rev. B 67, 045311 (2003).

[12] M. Pi, A. Emperador, M. Barranco, F. Garcias, K. Muraki, S. Tarucha, D.G. Austing, Phys. Rev. Lett. 87, 066801 (2001). 